\title{
Level degeneracy and temperature-dependent carrier distributions in self-organized quantum dots
}

\author{
K. Kim ${ }^{\mathrm{a})}$ and T. B. Norris \\ Center for Ultrafast Optical Science, Department of Electrical Engineering and Computer Science, \\ The University of Michigan, 2200 Bonisteel Boulevard, Ann Arbor, Michigan 48109-2099 \\ S. Ghosh, J. Singh, and P. Bhattacharya \\ Solid State Electronics Laboratory, Department of Electrical Engineering and Computer Science, \\ The University of Michigan, Ann Arbor, Michigan 48109-2122
}

(Received 4 December 2002; accepted 27 January 2003)

\begin{abstract}
Using femtosecond three-pulse pump-probe spectroscopy, we investigated the transparency condition for the ground and first excited states in self-organized $\mathrm{In}_{0.4} \mathrm{Ga}_{0.6}$ As quantum dots at different temperatures and wavelengths. The temperature-dependent behavior of the transparency condition is consistent with calculations using a multilevel model with a large density of states in the quantum-well reservoir. The twofold spatial degeneracy of the first excited state and the temperature dependence of the thermal equilibrium processes were experimentally observed. (C) 2003 American Institute of Physics. [DOI: 10.1063/1.1563732]
\end{abstract}

Self-organized quantum-dot (QD) active regions are being researched intensively for laser and amplifier devices because of their low threshold current density, ${ }^{1}$ reduced temperature sensitivity, ${ }^{2}$ and high differential gain. ${ }^{3}$ Because the temperature dependence of the optical gain results in reduced QD laser performance and efficiency at higher temperatures, the threshold currents in QD laser devices ${ }^{4-6}$ have been investigated to understand the thermal behavior of QD lasers. Although the models of quantum dots developed so far indicate a few confined discrete states in the dot and a continuum in the barrier region, real self-organized quantum dots are highly disordered with a complex electronic structure. It is, therefore, highly desirable to determine as completely as possible the actual level degeneracies of the confined states, and the effective densities of states (DOS) of the excited states, in the real self-organized QD system, since these are critical parameters which determine device performance. In this letter, we describe a method using femtosecond transient gain spectroscopy which provides accurate measurements of the transparency carrier density, and hence, the level degeneracies and DOS. The behavior of the temperature- and wavelength-dependent transparency condition measurements supports a multilevel model with a large density of states in the wetting layer, and confirms the theoretically predicted spatial degeneracy factor of 2 for the first excited state.

The $\mathrm{In}_{0.4} \mathrm{Ga}_{0.6} \mathrm{As}$ QD sample used in this work is an undoped heterostructure 7,8 with four layers of $\mathrm{In}_{0.4} \mathrm{Ga}_{0.6} \mathrm{As}$ quantum dots, separated by $2.5 \mathrm{~nm}$ GaAs barriers, grown by molecular beam epitaxy. These layers are sandwiched between two 0.1- $\mu \mathrm{m}$-thick GaAs layers and two outer $0.5 \mu \mathrm{m}$ $\mathrm{Al}_{0.3} \mathrm{Ga}_{0.7} \mathrm{As}$ carrier confinement layers. The structures are grown on (001) semi-insulating GaAs substrates, which are subsequently removed through selective etching to enable differential transmission (DT) measurements. The $\mathrm{In}_{0.4} \mathrm{Ga}_{0.6} \mathrm{As}$ dots are grown at $520^{\circ} \mathrm{C}$ while the rest of the sample is grown at $620^{\circ} \mathrm{C}$. Cross-sectional transmission

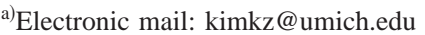

electron microscopy shows that the dots are pyramidal in shape with a base dimension of $14 \mathrm{~nm}$ and a height of $7 \mathrm{~nm}$. Atomic-force microscopy scans reveal a dot density of 5 $\times 10^{10} \mathrm{~cm}^{-2}$ per layer.

Band-structure calculations of individual QDs based on an eight-band $k \cdot p$ formalism predict several confined electronic levels and hole levels. ${ }^{9}$ The interband transition probabilities are high only for those transitions between electron and hole levels of the same quantum number. In real QD ensembles, these discrete levels are inhomogeneously broadened due to the size fluctuation of the dots. The first excited level in each dot has a twofold degeneracy due to the symmetry of the dot geometry, in addition to the double spin degeneracy. At low temperature, the excited state interband transition/E2H2/ is centered around $930 \mathrm{~nm}$, and the ground state transition $/ E 1 H 1 /$ is centered at $970 \mathrm{~nm}$. Figure 1(a) shows DT/T spectra (i.e., DT spectra normalized to the transmitted probe spectrum) of the sample measured using a 100 fs white-light probe pulse following excitation by an optical $(800 \mathrm{~nm})$ pump pulse for different pump fluences; saturation of the carrier population in the ground state is clearly observed.

Femtosecond three-pulse white-light pump and probe DT spectroscopy is performed using an $85 \mathrm{fs}, 3.5 \mu \mathrm{J}, 250$ kHz Ti:sapphire regenerative amplifier system. Carriers are injected by optically pumping the GaAs barrier region (800 $\mathrm{nm}$, "gain pulse") to establish a population inversion in QDs; the intensity of the gain pulse is adjusted so that the dots can be in the absorption or gain regime. ${ }^{10}$ Tunable pump and probe pulses are generated by spectrally filtering two single-filament white-light sources; a $10 \mathrm{~nm}$ bandwidth "pump pulse" is tuned to resonantly deplete (or generate) electron-hole pairs in the ground state or the first excited state by stimulated emission (or absorption) in the gain (or absorption) regime, after a 20 ps delay with respect to the gain pulse. The depletion (generation) of carriers by the pump pulse in ground state or the first excited state gives rise 

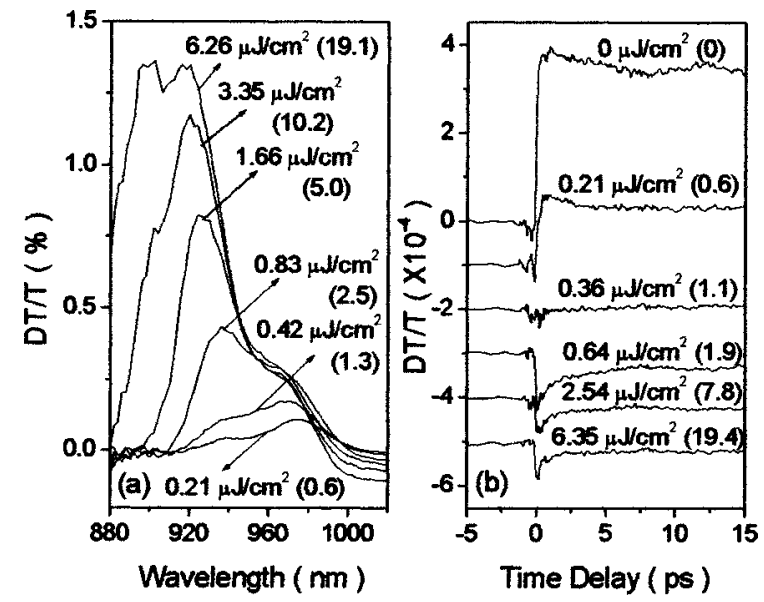

FIG. 1. (a) DT/T spectra measured with a broadband white-light probe pulse following injection of carriers into the barrier regions by an $800 \mathrm{~nm}$ "gain" pulse at a probe delay of $14 \mathrm{ps}$ for different gain pulse fluences, and (b) DT time scans measured with $970 \mathrm{~nm}$ pump and probe for different fluences of the gain pulse which injects the carriers at $t=-14 \mathrm{ps}$. The number of electron-hole pairs injected by the gain pulse normalized to the number of quantum dots in the focal volume is given in parentheses.

to a negative (positive) DT signal; this sign flip of the DT signal is the critical indication of gain in the QDs, and the transparency condition can be observed as zero DT signal. The pump pulse is fixed at a 20 ps time delay with respect to the gain pulse, because the carrier populations in the ground state and the excited state are saturated at this time. The pump pulse is mechanically chopped at $6 \mathrm{kHz}$, and the DT signal is measured using a lock-in amplifier; the probe DT is plotted as a function of delay following the pump pulse. The three pulses are focused on the QD sample near normal incidence; in this geometry we obtain the intrinsic single-pass transparency condition in QDs, uncomplicated by any propagation effects or device parasitics that one might have in a p-i-n waveguide structure. ${ }^{4-6}$

Figure 1(b) shows time-resolved degenerate pump-probe scans of the ground state $(E 1 H 1)$ DT signal for different gain-pulse fluence densities at $10 \mathrm{~K}$. The DT signal is positive (induced transmission) when the gain pulse fluence is zero, and becomes negative when the gain pulse exceeds the transparency fluence of $0.36 \mu \mathrm{J} / \mathrm{cm}^{2}(\sim 1.1 e-h$ pair per dot); this number is consistent with the data of Fig. 1(a), which shows that half of the $n=1$ states are full at this excitation density. The numbers of $e-h$ pairs per dot in parentheses in Fig. 1 are obtained from the measured spot size and power of the gain pulse using published values of the absorption coefficient $\left(\alpha \sim 12045 \mathrm{~cm}^{-1}\right)$ and reflectivity $(R \sim 0.3285) .{ }^{11}$

We measured the transparency condition for the ground and first excited states over the temperature range from 10 to $300 \mathrm{~K}$; the experimental carrier numbers per dot for zero gain are shown in Fig. 2(a). The transparency density is one electron-hole pair per dot in the ground state at low temperature (since there is a double spin degeneracy); the transparency density of the first excited state is observed to be 4 , which is exactly as expected for a spatial degeneracy of 1 in the ground state and 2 in the excited state. The transparency density exhibits a dramatic change around $100 \mathrm{~K}$, which indicates that at temperatures $>100 \mathrm{~K}$ thermal excitation to higher excited states and the continuum becomes dominant.
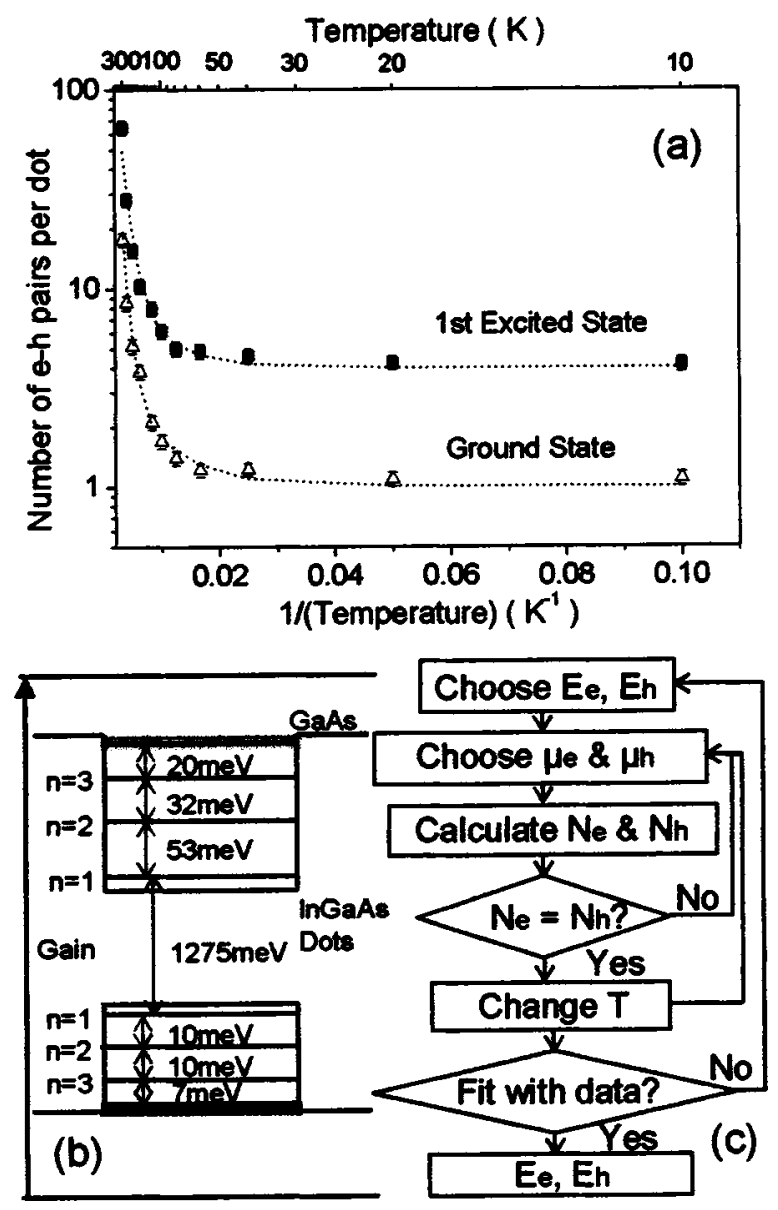

FIG. 2. $800 \mathrm{~nm}$ gain pulse was $20 \mathrm{ps}$ before the pump. (a) The transparency carrier numbers are measured for ground state (triangles) and first excited state (squares) in the range from 10 to $300 \mathrm{~K}$, and the dotted lines are calculations using a multilevel model with the energy levels in (b). The algorithm used in this calculation is shown in (c).

The temperature-dependent carrier dynamics at low carrier density also show similar behavior, and we developed a Monte Carlo simulation method which can explain our experimental data. ${ }^{12}$ (Note that these zero-gain measurements are averages over the pump's $10 \mathrm{~nm}$ bandwidth; thus, the reduction of threshold current within the $77-150 \mathrm{~K}$ range observed in threshold measurements of lasers ${ }^{5,6}$ is not seen in our experiments.)

The dotted lines in Fig. 2(a) are theoretical calculations using a model of discrete levels in the QD and subbands in the quantum-well $(\mathrm{QW})$ reservoir. Using a standard QW calculation of $0.2 \mu \mathrm{m}$ GaAs layer between two outer $0.5 \mu \mathrm{m}$ $\mathrm{Al}_{0.3} \mathrm{Ga}_{0.7} \mathrm{As}$ layers, the energy levels and densities of states of the QW reservoir were obtained. The numbers of subbands in the QW are 41, 84, and 36 for electrons, heavy holes, and light holes, respectively, and these QW reservoir states contribute to QD electron and hole distribution as following for the dot density of $\mathrm{n}_{\mathrm{QD}}$ with four layers:

$$
\begin{aligned}
N_{e}= & \sum_{i=1}^{3} \frac{g_{i}}{\exp \left[\left(E_{i, e}-\mu_{e}\right) / k_{B} T\right]+1}+\sum_{E_{r, e}} \frac{m_{e}^{*} k_{B} T}{4 \pi \hbar^{2} n_{\mathrm{QD}}} \\
& \times \ln \left\{1+\exp \left[\left(\mu_{e}-E_{r, e}\right) / k_{B} T\right]\right\},
\end{aligned}
$$




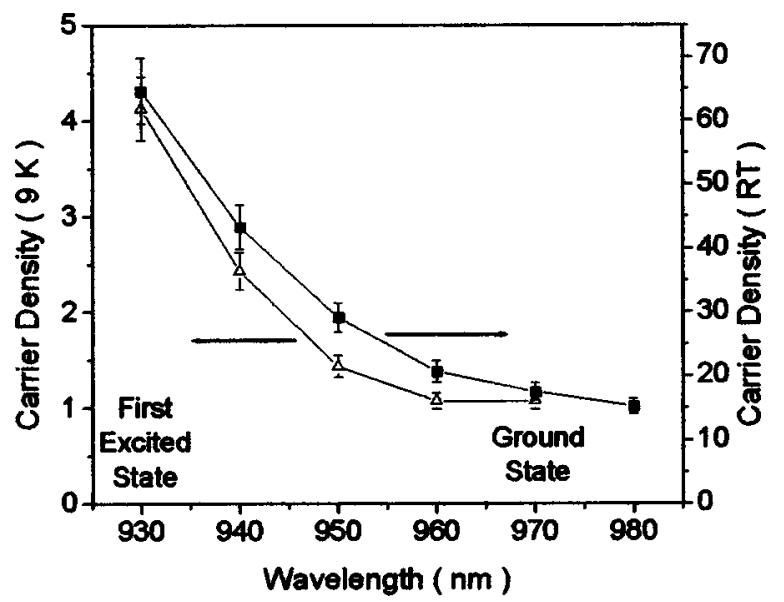

FIG. 3. $800 \mathrm{~nm}$ gain pulse was $20 \mathrm{ps}$ before the pump. The transparency carrier numbers were measured at $9 \mathrm{~K}$ (triangles) and $300 \mathrm{~K}$ (squares) in the spectral range from ground state to excited state.

$$
\begin{aligned}
N_{h}= & \sum_{i=1}^{3} \frac{g_{i}}{\exp \left[\left(\mu_{h}-E_{i, h}\right) / k_{B} T\right]+1}+\sum_{E_{r, \mathrm{hh}}} \frac{m_{\mathrm{hh}}^{*} k_{B} T}{4 \pi \hbar^{2} n_{\mathrm{QD}}} \\
& \times \ln \left\{1+\exp \left[\left(E_{r, \mathrm{hh}}-\mu_{h}\right) / k_{B} T\right]\right\}+\sum_{E_{r, \mathrm{hh}}} \frac{m_{\mathrm{lh}}^{*} k_{B} T}{4 \pi \hbar^{2} n_{\mathrm{QD}}} \\
& \times \ln \left\{1+\exp \left[\left(E_{r, \mathrm{hh}}-\mu_{h}\right) / k_{B} T\right]\right\} .
\end{aligned}
$$

$N_{e}$ and $N_{h}\left(\mu_{e}\right.$ and $\left.\mu_{h}\right)$ are the numbers (quasi-Fermi levels) of electrons and holes per dot, and $g_{i}$ is the state degeneracy including spin. From the zero-gain condition, we have $\mu_{h}$ $=\mu_{e}-E_{e}+E_{h}$. If we choose the energy levels of the electrons and holes $\left(E_{i, e}\right.$ and $\left.E_{i, h}\right), N_{e}$ and $N_{h}$ can be obtained with the constraint $N_{e}=N_{h}$ by varying $\mu_{e}$ and $\mu_{h}\left(=\mu_{e}\right.$ $-E_{e}+E_{h}$ from zero gain condition). We searched $E_{i, e}$ and $E_{i, h}$ for the best fit to the data, and found a model with three discrete energy levels inside QD with optical transitions at 973, 927, and $899 \mathrm{~nm}$, and the wetting layers above $881 \mathrm{~nm}$; these are consistent with the peaks of DT/T spectra in Fig. 1(a). The results of the fit are shown as the dotted lines in Fig. 2(a). In this and previous experiments we have shown that the dynamics ${ }^{12}$ and parameters such as the transparency condition in real self-organized quantum dot structures are driven in large part by the presence of a large density of states in the wetting layer and barrier region in the disordered system. This will have a major effect on any quantum-dotbased optoelectronic devices (interband or intersubband laser, mid-IR detector, etc.). One promising approach to reduce this problem is to use tunneling injection device which is injecting carriers into the quantum dot active region via phonon-assisted tunneling from the two-dimensional (2D) QW directly into the zero-dimensional (0D) QD ground state. $^{13}$

We also measured the wavelength-dependent transparency conditions of QDs in the spectral region from the ground state to first excited state at $9 \mathrm{~K}$ and room temperature (RT) as shown in Fig. 3. The transparency carrier densities around the ground state do not change in the spectral region from 960 to $980 \mathrm{~nm}$ at $9 \mathrm{~K}$, but do change at RT. As the carrier capture probabilities from the barrier region are approximately the same for different-sized dots, the zerogain densities are the same over the inhomogeneously broadened ground state, indicating there is no thermal equilibration process through the wetting layers or barrier at low temperature $(9 \mathrm{~K}) .^{14,15}$ The increase of transparency carrier numbers at the wavelength shorter than $950 \mathrm{~nm}$ is due to the increase of the distribution overlap with the first excited state. At temperatures above $100 \mathrm{~K}$ up to RT, the transparency densities increase dramatically due to thermal excitation. The variation in transparency density from 960 to 980 $\mathrm{nm}$ indicates thermal equilibrium at RT between different QDs (driven by thermal reemission into the barrier region and subsequent recapture).

In conclusion, we have measured the transparency condition for the ground and first excited states of undoped InGaAs QDs at different temperatures and wavelengths using a three-pulse pump-probe experiment. The spatial degeneracy factor 2 of the first excited state as well as the effect of temperature on the thermal equilibration processes is experimentally observed. The temperature-dependent behavior of transparency condition is consistent with our model calculation with a large density of states in the wetting layer and barrier region; this indicates that tunneling injection is a promising approach to avoid this limitation. ${ }^{13}$

The authors thank C. H. Schneider at Sandia National Laboratory for helpful discussion about theoretical modeling and K. Kamath and J. D. Phillips for their contributions. This work is supported by ARO Grant Nos. DAAH04-96-1-0414 and DAAG55-98-1-0419, the NSF through Grant No. ECS 9820129, and the AFOSR MURI.

\footnotetext{
${ }^{1}$ D. Bimberg, M. Grundmann, and N. N. Ledentsov, in Quantum Dot Heterostructures (Wiley, Chichester, 1998).

${ }^{2}$ Y. Arakawa and H. Sakaki, Appl. Phys. Lett. 40, 939 (1982).

${ }^{3}$ Y. Arakawa and A. Yariv, IEEE J. Quantum Electron. QE-22, 1887 (1986).

${ }^{4}$ G. Park, O. B. Shchekin, and D. G. Deppe, IEEE J. Quantum Electron. 36, 1065 (2000).

${ }^{5}$ A. Patane, A. Polimeni, M. Henini, L. Eaves, P. C. Main, and G. Hill, J. Appl. Phys. 85, 625 (1999).

${ }^{6}$ A. E. Zhukov, V. M. Ustinov, A. Y. Egorov, A. R. Kovsh, A. F. Tsatsulnikov, N. N. Ledentsov, and S. V. Zaitsev, Jpn. J. Appl. Phys., Part 1 36, 4216 (1997).

${ }^{7}$ T. S. Sosnowski, T. B. Norris, H. Jiang, J. Singh, K. Kamath, and P. Bhattacharya, Phys. Rev. B 57, R9423 (1998).

${ }^{8}$ J. Urayama, T. B. Norris, J. Singh, and P. Bhattacharya, Phys. Rev. Lett. 86, 4930 (2001).

${ }^{9}$ H. Jiang and J. Singh, Phys. Rev. B 56, 4696 (1997).

${ }^{10}$ K. Kim, J. Urayama, J. Singh, J. Phillips, and P. Bhattacharya, Appl. Phys. Lett. 81, 670 (2002).

${ }^{11}$ D. E. Aspnes, S. M. Kelso, R. A. Logan, and R. Bhat, J. Appl. Phys. 60, 754 (1986).

${ }^{12}$ J. Urayama, T. B. Norris, J. Singh, and P. Bhattacharya, Appl. Phys. Lett. 80, 2162 (2002).

${ }^{13}$ P. Bhattacharya, S. Ghosh, S. Pradhan, J. Singh, Z.-K. Wu, J. Urayama, K. Kim, and T. Norris (unpublished).

${ }^{14}$ L. V. Asryan and R. A. Suris, Semicond. Sci. Technol. 11, 554 (1996).

${ }^{15}$ H. D. Summers, J. D. Thomson, P. M. Smowton, P. Blood, and M. Hopkinson, Semicond. Sci. Technol. 16, 140 (2001).
} 